EPJ manuscript No.

(will be inserted by the editor)

\title{
MACRO results on atmospheric neutrino oscillations
}

\author{
G. Giacomelli ${ }^{1}$, A. Margiotta ${ }^{1}$ \\ For the MACRO Collaboration \\ e-mail: giacomelli@bo.infn.it, and e-mail: margiotta@bo.infn.it
}

Dipartimento di Fisica and INFN, I-40127 Bologna, Italy

Paper presented at the HEP EPS Conf., Aachen, Germany, July 2003

\begin{abstract}
The final results of the MACRO experiment on atmospheric neutrino oscillations are presented. The data concern different event topologies with average neutrino energies of $\sim 3$ and $\sim 50 \mathrm{GeV}$. Multiple Coulomb Scattering of the high energy muons was used to estimate the neutrino energy event by event. The angular distributions, the $L / E_{\nu}$ distribution, the particle ratios and the absolute fluxes all favour $\nu_{\mu} \rightarrow \nu_{\tau}$ oscillations with maximal mixing and $\Delta m^{2} \simeq 0.0023 \mathrm{eV}^{2}$. Emphasis is given to measured ratios which are not affected by Monte Carlo (MC) absolute normalization; a discussion is made on MC uncertainties.
\end{abstract}

PACS. 13.15. $+\mathrm{g} \nu$ interactions $-14.60 . \mathrm{Pq} \nu$ mixing - 96.40.De CR composition energy spectra $-96.40 . \mathrm{TV}$ $\nu$ and $\mu$.

\section{Introduction}

MACRO was a large area multipurpose underground detector 1] designed to search for rare events and rare phenomena in the penetrating cosmic radiation. It was located in Hall B of the underground Gran Sasso Lab at an average rock overburden of 3700 m.w.e.; it started data taking with part of the apparatus in 1989; it was completed in 1995 and was running in its final configuration until the end of 2000. The detector had global dimensions of $76.6 \times 12 \times 9.3 \mathrm{~m}^{3}$ and provided a total acceptance to an isotropic flux of particles of $\sim 10,000 \mathrm{~m}^{2} \mathrm{sr}$; vertically it was divided into a lower part, which contained 10 horizontal layers of streamer tubes, 7 of rock absorbers and 2 layers of liquid scintillators, and an upper part which contained the electronics and was covered by 1 layer of scintillators and 4 layers of streamer tubes. The sides were covered with 1 vertical layer of scintillators and 6 of limited streamer tubes.

MACRO detected upgoing $\nu_{\mu}$ 's via charged current interactions, $\nu_{\mu} \rightarrow \mu$; upgoing muons were identified with the streamer tube system (for tracking) and the scintillator system (for time-of-flight measurement). The events measured and expected for the three measured topologies, deviate from Monte Carlo expectations without oscillations, Fig 1 these deviations and the $L / E_{\nu}$ distribution point to the same $\nu_{\mu} \rightarrow \nu_{\tau}$ oscillation scenario [2-8], Fig. 2]

\section{Atmospheric neutrinos. Monte Carlo}

The measured data of Fig. 1] were compared with different MC simulations. In the past we used the neutrino flux computed by the Bartol96 group 9 and the GRV94 parton distribution. For the low energy channels the cross sections by $\mathrm{P}$. Lipari et al. were used; the propagation of muons to the detector used the energy loss calculation by Lohmann et al. The total systematic uncertainty in the predicted flux of upthroughgoing muons, was estimated at $\pm 17 \%$; this is mainly a scale error that does not change the shape of the angular distribution. The response of the detector to the passage of particles was simulated using GEANT3. A similar MC (Honda96) was used by the SuperK Collaboration 10 11.

Recently new improved MC predictions for neutrino fluxes were made available by the Honda [1] and FLUKA [12] groups. They include three dimensional calculations of hadron production and decays and of neutrino interactions, improved hadronic model and new fits of the primary cosmic ray flux. The two MC yield predictions for the non oscillated and oscillated $\nu_{\mu}$ fluxes equal to within few \% 8. The shapes of the angular distributions for oscillated and non oscillated Bartol96, new FLUKA and new Honda fluxes are the same to within few \%. The absolute values of our upthroughgoing muon data are about $25 \%$ above those predicted by the new FLUKA and Honda MC, Fig. 3. A similar situation is found in the new SuperK data 10. The high energy $\nu_{\mu}$ data thus suggest that the new Honda and FLUKA predictions are low, probably because of the used CR fit (the inclusion of the new ATIC Collab. measurements of primary CRs may improve the situation [13]). The evidence for neutrino oscillations is due mainly to the shape of the angular distribution and this is the same in all MCs. Also the ratios of the medium to high energy measurements and of the two different samples of 
low energy data are MC independent. Our low energy data suggest that the FLUKA normalization should be raised by about $12 \%$ at $E_{\nu} \sim 3 \mathrm{GeV}$.
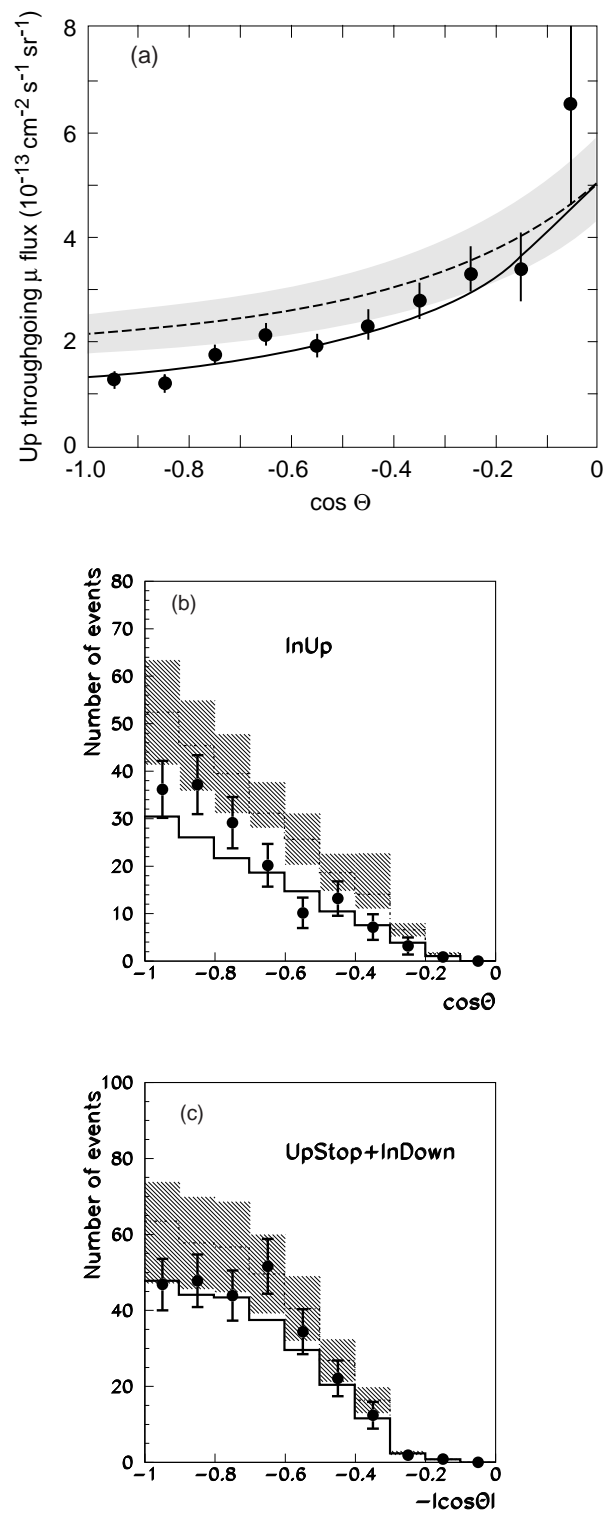

Fig. 1. Zenith distributions for the MACRO data (black points) for (a) upthroughgoing, (b) semicontained and (c) upstopping muons + down semicontained. The dashed line in (a) is the no-oscillation Bartol96 MC prediction (with a scale error band), (in b, c are the FLUKA MC; better fits are obtained with Bartol96); the solid lines refers to $\nu_{\mu} \rightarrow \nu_{\tau}$ oscillations with maximal mixing and $\Delta m^{2}=2.3 \cdot 10^{-3} \mathrm{eV}^{2}$ (see text).

\section{MACRO results on atmospheric neutrinos}

The upthroughgoing muons come from $\nu_{\mu}$ interactions in the rock below the detector; muons with $E_{\mu}>1 \mathrm{GeV}$ cross

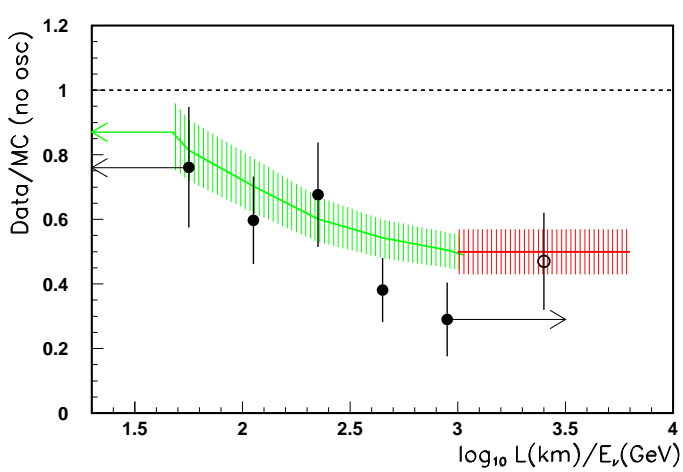

Fig. 2. Ratio (Data/MC Bartol96) versus the estimated $L / E_{\nu}$ for the upthrougoing muon sample (black circles) and the semicontained up- $\mu$ (open circle). The horizontal dashed line at Data/MC $=1$ is the expectation for no oscillations.

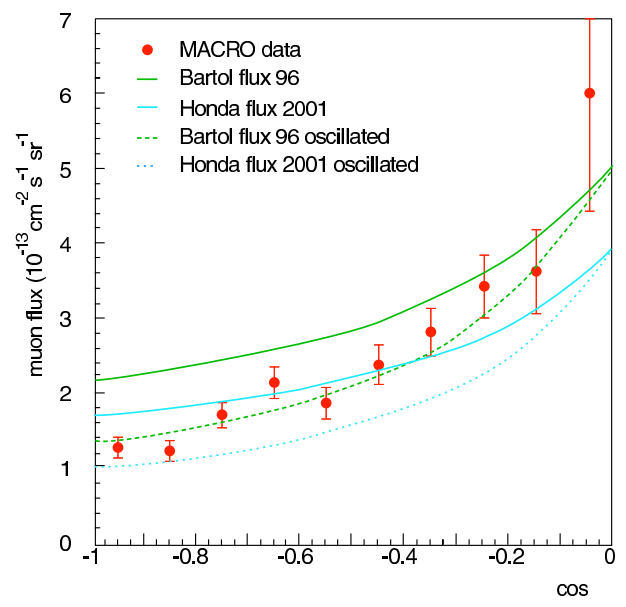

Fig. 3. Comparison of our measurements with the Bartol96 and the new Honda 2001 oscillated and non oscillated fluxes.

the whole detector. The corresponding $\nu_{\mu}$ 's have a median energy $\bar{E}_{\nu} \sim 50 \mathrm{GeV}$. Many possible systematic effects and backgrounds that could affect the measurements were studied 3 8. The data, Fig. 17, deviate in shape and in absolute value from the Bartol96 MC non oscillated predictions.

$\nu_{\mu} \rightarrow \nu_{\tau}$ versus $\nu_{\mu} \rightarrow \nu_{s}$. Matter effects would produce a different total number and a different zenith angle distribution of upthroughgoing muons. The ratio $R_{1}=$ Vertical/Horizontal $=N(-1<\cos \theta<-0.7) / N(-0.4<$ $\cos \theta<0)$ was used to test the $\nu_{\mu} \rightarrow \nu_{s}$ oscillation hypothesis versus $\nu_{\mu} \rightarrow \nu_{\tau}$ [2] 6] [6]. The $\nu_{\mu} \rightarrow \nu_{s}$ oscillations (with any mixing) are excluded at about $99.8 \%$ c.l. with respect to $\nu_{\mu} \rightarrow \nu_{\tau}$ oscillations with maximal mixing [8]. Oscillation probability as a function of the ratio $L / E_{\nu}$. $E_{\nu}$ was estimated by measuring the muon energy, $E_{\mu}$, by means of the muon Multiple Coulomb Scattering (MCS) in the rock absorbers in the lower MACRO. The space resolution achieved is $\simeq 3 \mathrm{~mm}$. For each muon, seven variables 
were given in input to a Neural Network (NN) trained to estimate muon energies with MC events of known input energy crossing the detector at different zenith angles. The distribution of the ratio $R=\left(\right.$ Data $\left./ M C_{\text {noosc }}\right)$ obtained by this analysis is plotted in Fig. [2 as a function of $\left(L / E_{\nu}\right)$ [7]. The data extend from $\left(L / E_{\nu}\right) \sim 30$ to $5000 \mathrm{~km} / \mathrm{GeV}$.

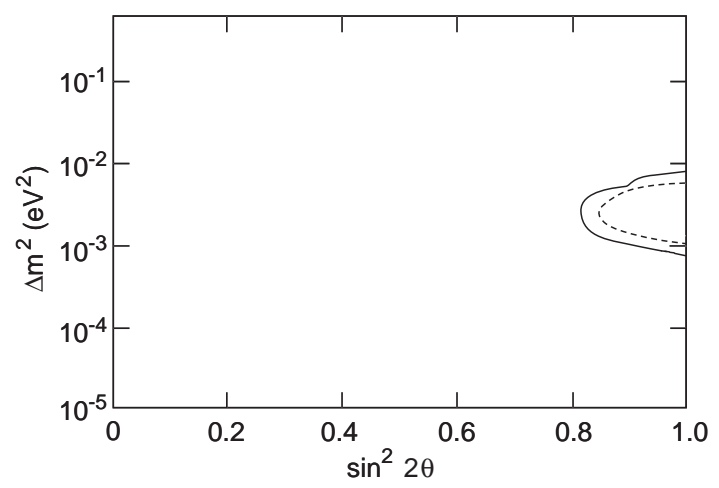

Fig. 4. Interpolated qualitative $90 \%$ C.L. contour plots of the allowed regions in the $\Delta m^{2}-\sin ^{2} 2 \theta$ plane for the MACRO data using only the ratios $R_{1}, R_{2}, R_{3}$ (outer continuous line) and using also the absolute values assuming the validity of the Bartol96 fluxes (dotted line).

The Internal Upgoing (IU) muons come from $\sim 3 \mathrm{GeV}$ $\nu_{\mu}$ 's interacting in the lower apparatus. Compared to the no-oscillation prediction there is a reduction in the flux of these events, without distortion in the shape of the zenith distribution, Fig. 10. The MC predictions for no oscillations in Figs. П and Mre the dashed lines with a $21 \%$ systematic band. At these energies the ratios DATA/MC are 0.67 for FLUKA and 0.54 for Bartol96.

The Upstopping (UGS) muons are due to $\sim 3 \mathrm{GeV} \nu_{\mu}$ 's interacting below the detector, yielding upgoing muons stopping in the detector. The Semicontained Downgoing (ID) muons are due to $\nu_{\mu}$-induced downgoing $\mu$ 's with vertex in the lower MACRO. The two types of events are identified by topological criteria. The upgoing $\nu_{\mu}$ 's should have oscillated completely, while the downgoing $\nu_{\mu}$ do not.

\section{Determination of the oscillation parameters.}

In the past, in order to determine the oscillation parameters, we made fits to the shape of the upthroughgoing muon zenith distribution and to the absolute flux compared to the Bartol96 MC prediction. The other data were only used to verify the consistency and to make checks. The result was $\Delta m^{2}=0.0025 \mathrm{eV}^{2}$ and maximal mixing [6] [3. Later, also the $L / E_{\nu}$ distribution was considered 7.

In order to reduce the effects of possible systematic uncertainties in the MC we recently used the following three independent ratios 8 and we checked that FLUKA, Honda and Bartol96 Monte Carlo simulations yield the same predictions to within $\sim 5 \%$.

(i) High Energy Data: zenith distribution ratio: $R_{1}=$ $N_{\text {vert }} / N_{\text {hor }}$

(ii) High Energy Data, neutrino energy measurement ratio: $R_{2}=N_{\text {low }} / N_{\text {high }}$

(iii) Low Energy Data:

Ratio $R_{3}=(\text { Data } / M C)_{I U} /(\text { Data } / M C)_{I D+U G S}$.

With these ratios, the no oscillation hypothesis has a probability $P \sim 3 \cdot 10^{-7}$ and is thus ruled out by $\sim 5 \sigma$. By fitting the three ratios to the $\nu_{\mu} \rightarrow \nu_{\tau}$ oscillation formulae we obtain $\sin ^{2} 2 \vartheta=1, \Delta m^{2}=2.3 \cdot 10^{-3} \mathrm{eV}^{2}$ and the allowed region indicated by the solid line in Fig. 4 .

There is a good consistency between the old and new methods.

If we use the Bartol96 flux we may add to the ratios (i) - (iii) the information on the absolute flux values of the

(iv) high energy data (systematic scale error of $\gtrsim 17 \%$ ) $R_{4}=$ $N_{\text {meas }} / N_{M C \text { Bartol }}$.

(v) low energy semicontained muons, with a systematic scale error of $21 \%, R_{5} \simeq N_{\text {meas }} / N_{M C \text { Bartol }}$.

These informations reduce the area of the allowed region in the $\Delta m^{2}-\sin ^{2} 2 \theta$ plane, as indicated by the dashed line in Fig. 14 The limit lines represent smoothed interpolations and are qualitative. The final MACRO $\Delta m^{2}$ is $2.3 \cdot 10^{-3} \mathrm{eV}^{2}$.

We would like to acknowledge the cooperation of the members of the MACRO collaboration.

\section{References}

1. S. Ahlen et al., Nucl. Instr. Meth. Phys. Res. A324(1993)337; M. Ambrosio et al., Nucl. Instr. Meth. Phys. Res A486(2002)663.

2. G. Giacomelli et al., hep-ex/0211035 hep-ex/0210006

Modern Phys. Lett. A18(2003)2001; hep-ex/0407023

3. M. Ambrosio et al., Astrop. Phys. 9(1998)105; "Status report 2001", hep-ex/0206027

4. S. Ahlen et al., Phys. Lett. B357(1995) 481.

5. M. Ambrosio et al., Phys. Lett. B434(1998)451; Phys. Lett. B478(2000)5; G. Giacomelli et al., hep-ph/9901355 hep-ex/0201032

6. M. Ambrosio et al., Phys. Lett. B517(2001)59.

7. M. Ambrosio et al., Nucl. Instr. Meth. Phys. Res. A492(2002)376; Phys. Lett. B566(2003)35;

8. M. Ambrosio et al., Eur. Phys. J. C36(2004)357.

9. V. Agrawal et al., Phys. Rev. D53(1996)1314.

10. Y. Hayato, "Status of the Super-K and the K2K experiments", HEP EPS Conf., Aachen, (2003).

11. M. Honda et al., Phys. Rev. D64(2001)053011; Phys. Rev. D52(1995)4995.

12. G. Battistoni et al., Astrop. Phys. 19(2003)269; "Erratum", Astrop. Phys. 19(2003)291.

13. R. Battiston, Rapporteur talk; T. Montaruli, Rapporteur talk, ICRC 2003, Tsukuba, Japan. 